\title{
La lutte pour la culotte, un topos iconographique des rapports conjugaux ( $\mathrm{XV}^{\mathrm{e}}-\mathrm{XIX}^{\mathrm{e}}$ siècles)
}

"The struggle for the breeches", an iconographical topos( $15^{\text {th }}-19^{\text {th }}$ century)

\section{Christiane Klapisch-Zuber}

\section{OpenEdition}

\section{Journals}

Édition électronique

URL : https://journals.openedition.org/clio/10331

DOI : 10.4000/clio.10331

ISSN : 1777-5299

Éditeur

Belin

Édition imprimée

Date de publication : 31 décembre 2011

Pagination : 203-218

ISBN : 978-2-8107-0170-4

ISSN : $1252-7017$

Référence électronique

Christiane Klapisch-Zuber, «La lutte pour la culotte, un topos iconographique des rapports conjugaux (xve-xIx ${ }^{\mathrm{e}}$ siècles) », Clio. Femmes, Genre, Histoire [En ligne], 34 | 2011, mis en ligne le 31 décembre 2013, consulté le 22 avril 2022. URL : http://journals.openedition.org/clio/10331 ; DOI : https://doi.org/ 10.4000/clio. 10331 


\section{Documents}

\section{La lutte pour la culotte, un topos iconographique des rapports conjugaux ( $\mathrm{XV}^{\mathrm{e}}-\mathrm{XIX}^{\mathrm{e}}$ siècles)}

$\mathrm{La}$ « lutte pour la culotte » a fait rire bien des générations au cours des siècles. Ce thème iconographique, pourtant, voulait aussi faire réfléchir sur l'ordre du monde et la hiérarchie fondamentale qui régentait les rapports entre les sexes. Consacré au Moyen Âge par les représentations visuelles plus encore que par la littérature, il critique en effet l'appropriation par les femmes de l'autorité 'naturelle' des hommes. Il traverse le Moyen Âge, s'épanouit à la Renaissance et perdure jusqu'à la fin du XIXe siècle sous des formes diverses. Le lien qui unit ses variantes peut être lu dans la perspective psychanalytique de la peur masculine universelle devant la castration et la dévirilisation. Mais l'historien, pour l'interpréter, ne peut ignorer le contexte social et l'environnement visuel dans lequel ces images ont pris sens et ont été regardées par les contemporains.

Depuis la fin du Moyen Âge les supports les plus variés et des œuvres de toutes dimensions ont diffusé le thème: depuis les «drôleries » couvrant les marges de manuscrits ${ }^{1}$ jusqu'aux fresques publiques ou curiales en passant par les «miséricordes » des chœurs

1 Randall 1966 ; Camille 1992 et 1998. 
d'église ${ }^{2}$ et par le décor d'objets destinés à l'usage domestique. Les premières gravures de grande diffusion, au XVI $\mathrm{X}^{\mathrm{e}}$ siècle, relaient ces emplois dispersés, l'estampe ultérieure se délecte du motif et les images d'Épinal l'introduisent dans les foyers du XIX siècle.

$\mathrm{La}$ «lutte pour la culotte» dit en termes plus voilés ce que d'autres images peuvent aussi dire de façon très crue et en s'en divertissant - parce que ce qui touche au sexe peut être exprimé à condition de faire rire -, à savoir la place centrale de la sexualité dans les passions et les aspirations humaines ${ }^{3}$, mais c'est pour la critiquer lorsqu'elle enfreint les normes sociales prévalentes. Elle met donc en cause les rapports de genre, et plus particulièrement la place respective des hommes et des femmes dans la sphère sociale ou domestique, et ce faisant elle dispense à ses spectateurs une leçon essentiellement normative.

Les représentations médiévales qui se rapportent à la sexualité donnent très souvent à voir la nudité des femmes et des hommes non pas dans leur entier, mais par un détail. Elles choisissent de dire et de montrer les organes génitaux de façon tout à fait explicite en les détachant de leur porteur ou porteuse naturel-le. Ainsi, les soties, fabliaux et autres moralités mettent volontiers en scène l'appareil sexuel masculin ou féminin ${ }^{4}$. Les marges d'un célèbre manuscrit du Roman de la Rose, souvent commentées, fourmillent de thèmes " érotiques $»^{5}$. Dans un autre registre, des insignes comme les badges de pèlerinage comportent souvent une représentation de l'organe génital mâle ou femelle et sont gaillardement arborés par les voyageurs et les pèlerins: depuis l'Antiquité de telles images ont valeur protectrice et apotropaïque. Fleurissent ainsi des images surprenantes sur le couvre-chef ou le manteau de personnes par ailleurs animées par la dévotion, par exemple trois phallus portant une vulve couronnée (1375-1425), des pantalons promenant des phallus, des femmes qui en brouettent des monceaux 6 .

2 Les miséricordes sont des sortes d'appuie-fesses sur lesquels les religieux de chœur pouvaient se reposer durant les longs offices, Bureau 1995.

3 Jones 1994.

4 Fabliaux érotiques 1993 : 137-153 ; Contes pour rire 1977: 138-140 et 58-63 («Les quatre souhaits de saint Martin ») ; Bloch 1986.

5 Huot 1993 ; Camille 1998.

6 Beuningen \& Koldeweij 1993. 
De ces images qui ont assurément, pour les premières, valeur critique (la paillardise des moines et des nonnes est dénoncée) et, pour les secondes, valeur magique (les badges de pèlerins les protègent des dangers du voyage), on peut aussi penser qu'elles renvoient également à un registre de représentations symboliques et morales. Plusieurs auteurs ont montré que, si l'on isole ces images de leur contexte, on ignore la pertinence de leur leçon. Une miséricorde prend toute sa signification si on l'étudie en rapport avec ses voisines; de même un grotesque marginal doit être mis en relation avec le texte et l'illustration principale auxquels il apporte fréquemment un contrepoint sur le mode de la dérision ${ }^{7}$. Les badges sexuels eux aussi insistent sur la carnalité de leurs porteurs au moment même où ils sont en quête d'un lieu de prière et de repentir.

D'autres images suggèrent également que la représentation de situations en apparence les plus rivées à la 'chair' comporte une intention éthique ou spirituelle ${ }^{8}$. Moins connu que la «lutte pour la culotte », le thème de l'«arbre aux pénis » a récemment été reporté à la lumière après qu'on ait découvert dans une petite ville de la Maremme siennoise, Massa Marittima, une fresque ornant la fontaine publique de la ville, représentant un grand arbre dont les fruits sont des phallus que se disputent les femmes ${ }^{9}$. Rapprochée d'autres décors castraux ou domestiques, la leçon morale de ces "arbres aux pénis » comporte à l'évidence une mise en garde contre les dangers de la conception courtoise de l'amour ${ }^{10}$. Or plusieurs de ces images présentent une vision contrastée de la cueillette: paisible d'un côté, belliqueuse de l'autre. Des femmes qui se disputent violemment le phallus, voilà qui avertit des dangers que leur prétention sexuelle fait courir à l'homme et à la société. Si l'une d'elles ou plusieurs d'entre elles prétendent se l'approprier, on peut s'attendre aux pires désordres et à la ruine d'une société policée, naturellement contrôlée par les hommes.

Un thème connexe, "L'arbre aux galants", dont tout porte à penser qu'il dérive de l'arbre aux pénis, concerne le choix du

\footnotetext{
7 Bureau 1995.

8 Je me permets de renvoyer à Klapisch-Zuber 2010.

9 Bagnoli 2000 ; Ferzoco s.d.

10 Wolter-von dem Knesebeck 2005.
} 
conjoint ${ }^{11}$. Le présupposé qu'il comporte (et la critique qui en découle) est qu'abandonner aux femmes l'initiative du choix de leur conjoint les portera à privilégier non pas l'époux le plus approprié au maintien de l'ordre social mais l'homme le plus désirable. Leur quête d'un galant prélude alors à un désordre qui va s'exprimer par leur rivalité, source de disputes, par la prédominance du désir passager sur le lien durable et, dans le meilleur des cas - lorsque cette quête d'un partenaire aboutira au mariage - par le mauvais appariement final des conditions des conjoints et par la tension des rapports conjugaux qui en résultera. Les femmes sont de dangereuses trouble-fête, qui dévoient les processus normaux de l'alliance et de la transmission des richesses. L'estampe de l'époque moderne à son tour va proposer les mises en garde nécessaires en actualisant l'arbre aux pénis médiéval et en en adoucissant les expressions les plus crues pour le rendre plus conforme aux impératifs de décence imposés par la Contre Réforme. C'est dans ce vaste éventail d'images se contaminant les unes les autres qu'il faut donc placer le thème de la « lutte pour la culotte ».

\section{Porter la culotte}

Les premiers exemples de la «lutte pour la culotte » apparaissent vers le milieu du XIII ${ }^{\mathrm{e}}$ siècle ${ }^{12}$. Ils lénifient la crudité des images des rapports entre hommes et femmes que les artistes médiévaux évoqués présentaient ironiquement à travers le désir sexuel brut des femmes, en transférant le problème général de l'autorité de l'homme sur la femme dans la sphère conjugale ou dans les rapports internes au couple. Dans ce cadre domestique, le phallus médiéval est remplacé par un objet symbolique, le vêtement intime de l'homme: les "braies», culotte ou chausses, présentées comme le signe métonymique de son autorité. L'affrontement d'une ou de plusieurs femmes autour d'un pantalon masculin semble proposer une version métaphorique, et adoucie, de l'appropriation féminine individuelle du phallus, emblème sexuel et symbole de l'autorité masculine. Ce thème iconographique opère un déplacement évident du contenu vers le

\footnotetext{
11 Klapisch-Zuber 2010.

12 Beaumont-Maillet 1984 ; Niccoli 1981 ; Metken 1996.
} 
contenant ${ }^{13}$. Le pouvoir masculin se cristallise ainsi pour des siècles dans un objet signifiant, qui a l'avantage d'offrir plus de possibilités de représentation que l'organe sexuel représenté au Moyen Âge, désormais banni de l'estampe.

«Porter la culotte» est une expression dont on trouve des occurrences dès le XIII ${ }^{\mathrm{e}}$ siècle dans les fabliaux voire dans les prêches qui reprennent des thèmes ou des termes populaires en guise d'exempla. Par exemple, le fabliau de Sire Hain et Dame Anieuse met en scène un couple bagarreur où la femme est ramenée à la discipline maritale à l'issue d'un combat autour des braies de l'homme; ou le fabliau des Quatre soubaits de saint Martin où la femme d'un vilain normand "chauçoit les braies " ${ }^{14}$. Mais il convient de distinguer entre les «disputes pour la culotte » qui opposent un homme et une femme et celles qui représentent un affrontement entre plusieurs femmes autour d'une culotte. Le second thème a connu une fortune plus courte que la première, mais il présente une similitude plus forte avec les images de l'arbre aux pénis.

\section{Batailles de femmes}

Parmi les plus anciennes de ces images d'une bataille de femmes, on trouve deux gravures. L'une est attribuée au graveur florentin Baccio Baldini (fig. 1) et l'autre à un artiste allemand anonyme - jadis appelé «le Maître de 1464 » et aujourd'hui désigné comme le «Maître des Banderoles » (fig. 2). Elles montrent l'empoignade d'une douzaine de femmes se battant pour s'emparer de cette partie du vêtement masculin'15. S'agit-il d'un jeu, ordonné par la Folie, dont les représentants figurent dans les deux gravures? Le fou médiéval est réputé ne pas porter ce sous-vêtement et l'homme réduit à la perte de ses braies tombe à son niveau : il devient fou lui-même, ou doit être considéré comme un fou. Le jeu des femmes est donc un jeu dangereux pour l'homme et, dans la version de Baldini (fig. 2), la Mort fait pendant à un fou à l'arrière-plan.

\footnotetext{
13 Sur les variantes de la culotte représentée aux XVe-XVI e siècles, Jaritz 1992.

14 Bureau 1995.

15 Lippmann 1886 : ill. p. 74-75.
} 


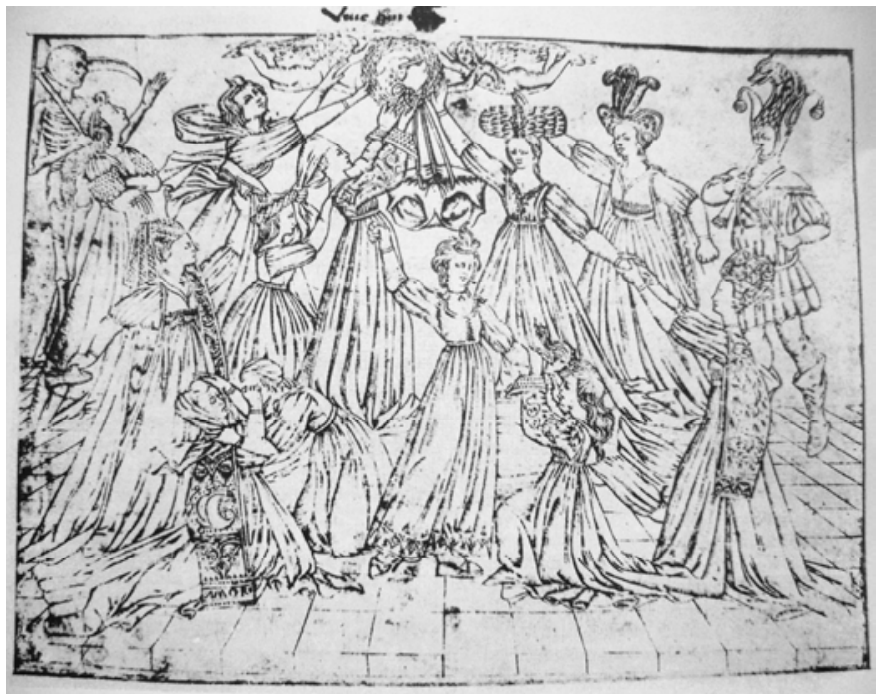

1. Baccio Baldini, Lutte pour la culotte. Florence, av. 1464. Munich, Staatliche Graphische Sammlungen

(d'après Zucker 1980, p. 252).

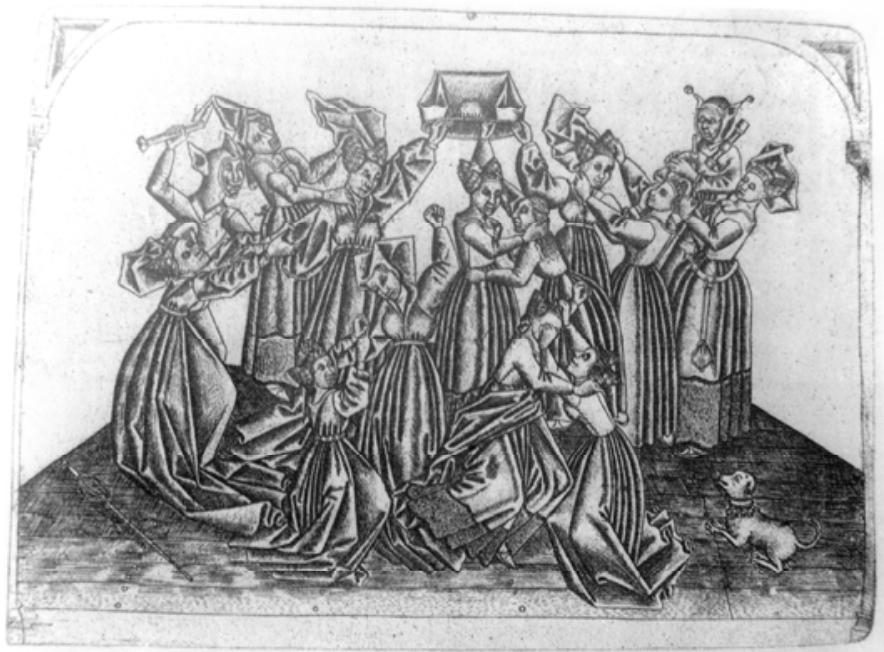

2. Maître des Banderoles, Lutte pour la culotte. Pays-Bas, v. 1464. Berlin, Kupferstichkabinett (d'après Holstein 1949, vol. 12 : 65). 
L'un des premiers à s'être interrogé sur les origines et les significations de ces gravures du Xve siècle a été Aby Warburg au tournant du XIX siècle $^{16}$. Dans la veine de l'iconologie naissante, il a voulu y reconnaitre un passage d'Isaïe : le prophète y clame que la Jérusalem corrompue comptera sept femmes pour un homme, et que ces femmes extorqueront à l'infortuné survivant sa protection et son nom pour sauver leur propre honneur :

Et sept femmes s'arracheront un homme, en ce jour-là, en disant: 'Nous mangerons notre pain, nous mettrons notre propre manteau, laisse-nous seulement porter ton nom. Ôte notre déshonneur' ${ }^{17}$.

Le thème semble avoir été populaire dans le nord de l'Europe, en Norvège par exemple et en Allemagne, où il a donné matière à des variations décoratives et pris place dans des réjouissances festives ou carnavalesques ${ }^{18}$. Selon l'hypothèse de Warburg reprise de Lippmann, premier éditeur de ces deux gravures, c'est d'Allemagne qu'il serait venu en Italie, transmis peut-être par Hartmann Schedel, le médecin humaniste auteur de la célèbre Chronique de Nuremberg illustrée (1493), qui possédait dans sa bibliothèque la gravure du Maitre des banderoles et avait des liens étroits avec l'Italie ${ }^{19}$.

Si le lien direct entre le texte d'Isaïe et les premières gravures de La lutte pour la culotte semble fragile, l'estampe ultérieure a cependant peut-être repris de la Bible le nombre des sept femmes, évidemment préféré par les artistes de la Renaissance aux douze mégères de Baldini et du "Maitre des banderoles" allemand (fig. 3). Mais, que les femmes soient sept ou douze, l'enjeu sexuel du combat est souligné par la frénésie qu'elles mettent à s'arracher l'objet convoité. Toutes les

16 Warburg 1998. L'iconographie de Warburg relative au thème est dans Der Bilderatlas Mnemosyne, 2000, Tafel 21-25.

17 Is. $4,1$.

18 Thème attesté au nord de l'Europe par la trouvaille faite par Warburg d'un coffre peint norvégien du XVII ${ }^{e}$ siècle, mais thème déjà repéré dans la comédie carnavalesque en Allemagne; Warburg 1998: 174-175. Sur les interprétations dans l'art populaire des XVIII'-XIXe siècles, voir Metken 1996 : 122-137.

19 Warburg $1998: 180$. 
versions les plus anciennes du thème font état de leur rivalité féroce et traduisent leur humeur batailleuse en privilégiant aux $\mathrm{XV}^{\mathrm{e}}$ et $\mathrm{XVI}^{\mathrm{e}}$ siècles les batailles de groupe ${ }^{20}$.

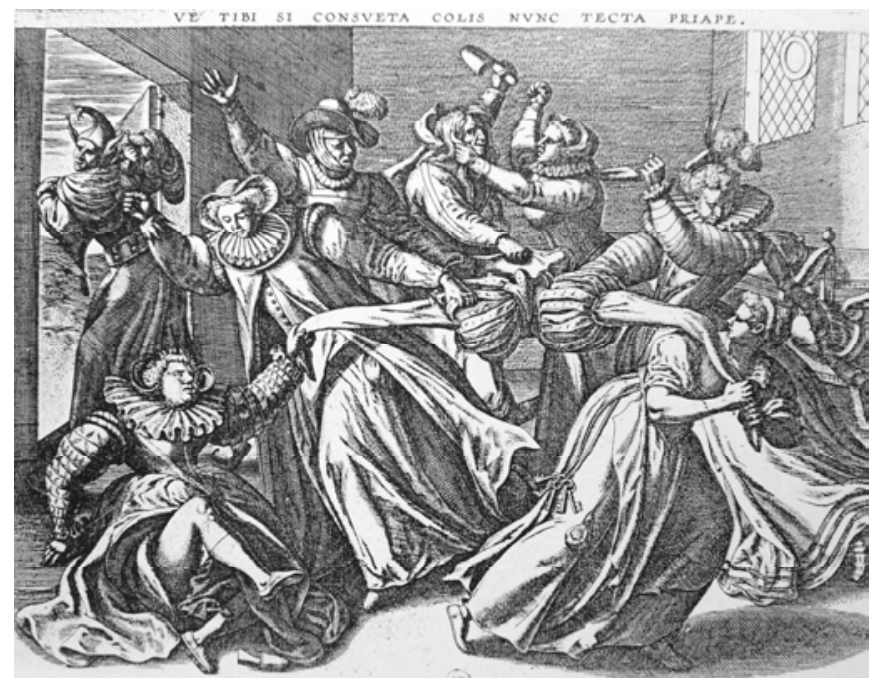

3. Anonyme, Combat de sept femmes pour la culotte. Paris, fin du XVI $\mathrm{s}$., Paris, BNF (d'après Hébert 1982 : 652).

\section{Le combat conjugal aux $\mathrm{XVII}^{\mathrm{e}}$-XIX ${ }^{\mathrm{e}}$ siècles}

L'affrontement de deux individus, de sexe différent, occupe par la suite le devant de la scène et domine l'estampe jusqu'au XIXe siècle. La dispute d'un homme et d'une femme autour d'une culotte est en effet l'autre version du thème. Des textes de fabliaux en offrent les prémisses. Dès le XIVe siècle on en trouve des représentations figurées dans les marginalia de manuscrits français ou flamands ${ }^{21}$. Le support le plus fréquent, dans ces régions du Nord, est cependant la "miséricorde", une commodité multipliée au Xve siècle, où les 'ymagiers' se plaisent à le sculpter ${ }^{22}$, invitant de la sorte chanoines et religieux à écraser l'arrogance féminine.

20 Sur les images de la violence des femmes, Matthews Grieco 1991 : 328-342.

21 Bureau 1995.

22 Kraus 1986 ; Bureau 1995. 
Cette «lutte pour la culotte» entre un homme et une femme renvoie évidemment au contexte conjugal, car où, sinon dans l'enceinte familiale, hommes et femmes pouvaient-ils se disputer l'autorité ? Elle croise là cependant un autre thème moralisant plus général, et largement répandu. Pour illustrer la soumission irrecevable de l'homme à la femme, les artistes du Moyen Âge ou de la Renaissance ont retenu l'histoire, alors bien connue et appréciée, d'Aristote chevauché par Phyllis, une redoutable séductrice aux charmes de laquelle le philosophe aurait succombé comme n'importe quel homme censément moins sage que lui (fig. 4). On représente aussi pour illustrer la même situation Samson perdant, sous les ciseaux de Dalila, ses cheveux et donc sa force et sa virilité. Au fil du temps, la dispute pour la culotte cohabite également avec un thème en vogue depuis la fin du XIVe siècle, celui du mari malmené et humilié par son épouse, qui forme comme la suite inéluctable d'une rivalité entre les sexes où la femme l'emporterait (fig. 5).

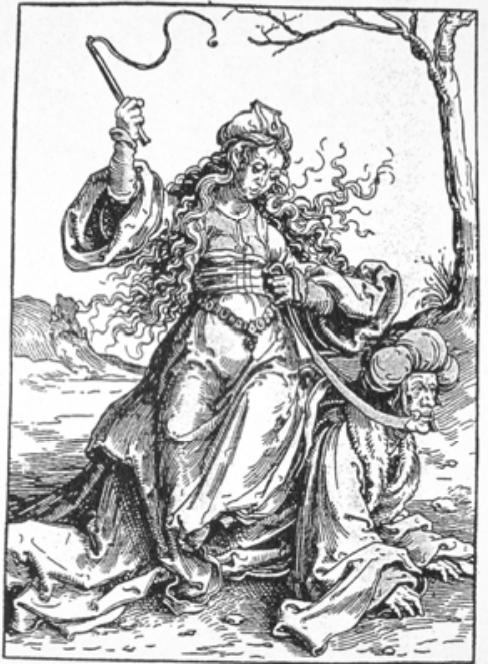

4. Anonyme, Aristote et Phyllis. Allemagne, début XVIe s. (d'après Grand-Carteret 19271928 , t. I, pl. XIV).

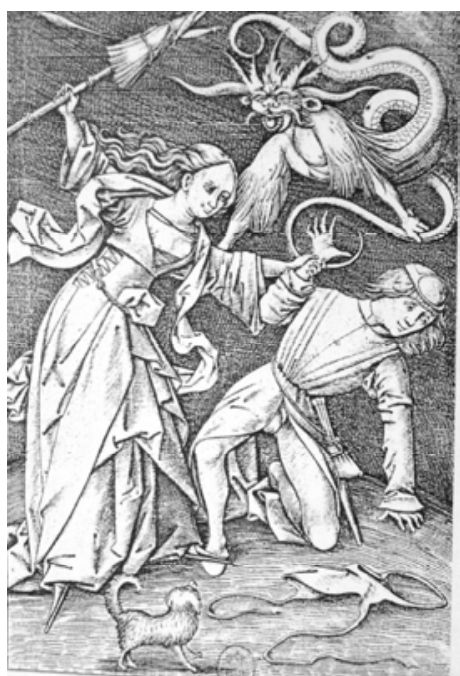

5. Israhel van Meckenem, La dispute pour la culotte, 1480. Paris, BNF, Estampes (d'après Beaumont-Maillet 1984 : 14). 
Une intéressante variante plus tardive de la lutte pour la culotte, au début du $\mathrm{XVII}^{\mathrm{e}}$ siècle, montre encore ce redoutable et prévisible enchaînement des faits : l'homme, qui s'est laissé priver de son attribut vestimentaire, assiste piteusement au combat enragé des femmes. Un graveur anglais de la seconde moitié du XVII ${ }^{e}$ siècle, John Smith, met ainsi en scène quatre femmes se disputant la culotte dont elles ont dépouillé un pauvre homme. Le lien entre la subversion de l'autorité masculine et la prétention des femmes à une sexualité autonome est expressément établi dans une autre variante où le combat des femmes coexiste avec le motif du mari dominé. Un dessin d'Augustin Braun, un artiste colonais du début du XVII ${ }^{\mathrm{e}}$ siècle, présente au premier plan la victoire de la femme qui enfile le pantalon d'un mari consentant et repentant, en chemise à ses pieds; dans un lointain arrière-plan, on distingue les sept femmes qui se livrent, à grands coups d'instruments ménagers, à la classique mêlée autour d'une culotte ${ }^{23}$. Dans cette version qui joue sur les contiguiltés des thématiques, la compétition féminine qui au fond de l'image se réfère au vieux modèle de l'avidité sexuelle des femmes, apparaît comme le prélude nécessaire à la perversion inéluctable du rapport conjugal. La quenouille, emblématique du destin 'naturel' de la femme, est ici jetée à terre, prête à être ramassée par le mari châtré par la perte de ses braies. Quant à lui, Israhel van Meckenem (vers 1480) en fait l'arme brandie par l'épouse dominatrice et ce sont les braies qui traînent sur le sol (fig. 5).

Dans les «Mondes à l'envers» imprimés du XVI ${ }^{\mathrm{e}}$ au XIX ${ }^{\mathrm{e}}$ siècle, une vignette présente inévitablement cet archétype du renversement du monde: la suprématie de l'épouse sur le mari. Et là encore la quenouille est bien souvent l'attribut de l'homme humilié par sa femme. Une telle inversion des rôles figure le bouleversement de l'ordre 'naturel' au même titre que celle du lièvre pourchassant le renard, du cavalier portant sa monture, du bœuf conduisant la charrue attelée au laboureur, des lapins faisant rôtir le chasseur et ses chiens etc. - tous motifs bien connus des «mondes à l'envers", depuis le temps lointain des marges de manuscrits médiévaux.

La bataille confuse qui se poursuit autour d'un misérable pantalon comporte à l'évidence la critique du désordre social que la victoire des

23 Vey 1964. 
femmes sur le sexe fort ne peut manquer d'introduire ${ }^{24}$. Mais, simultanément à leur abandon ou à la relégation à l'arrière-plan du vieux motif de la lutte féminine collective, les estampes modernes réduisent l'affrontement à celui des époux autour de la culotte du mari. Le combat est alors situé dans un espace moins abstrait, souvent installé de façon insistante dans la maison du couple, sous l'œil goguenard et le contrôle des voisins qui guettent l'occasion d'un beau charivari ${ }^{25}$ (fig. 6). Car si le désordre qu'engendre l'abus de pouvoir tenté par la femme à l'endroit de son maître légitime s'est replié dans le cadre familial, il n'en est pas moins délétère et des gravures ou des images d'Épinal le montrent parfois impliquant, pire : pervertissant tout l'entourage du couple, les enfants, les animaux domestiques...

\section{LA GRANDE QUERELLE DU MENAGE.}

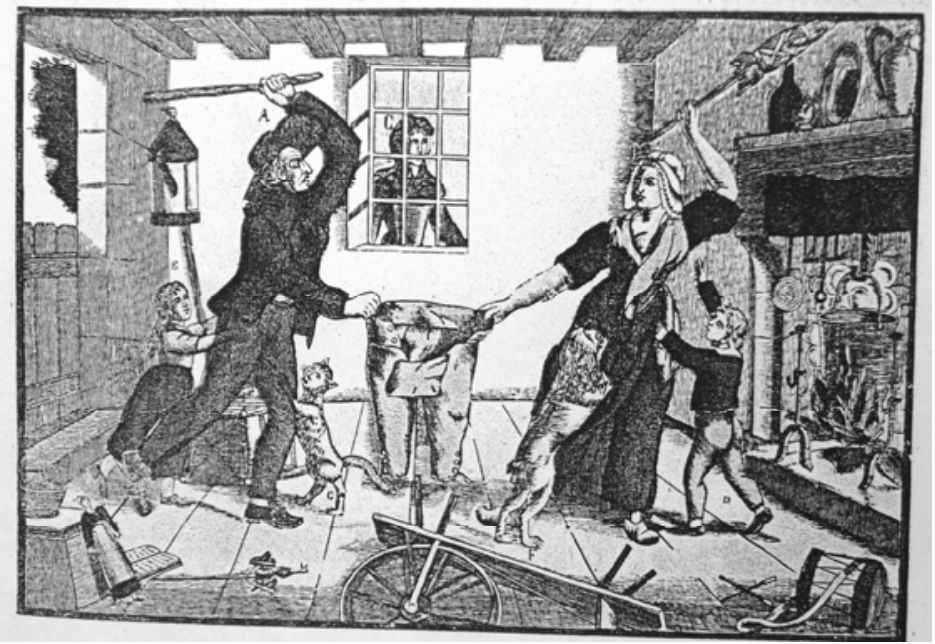

6. Anonyme, La grande querelle du ménage. Épinal, Pellerin, XIX ${ }^{\mathrm{e}} \mathrm{s}$.

(d'après Beaumont-Maillet 1984 : 19, ill. 11).

24 Beaumont-Maillet 1984 : 14-22, fig. 13 (fin du XVI s.), fig. 14 (v. 1635), fig. 15 (mil. du XVII s.).

25 Ibid. : fig. 4-12. Cf. Niccoli 1981. 
Transparente métaphore du phallus, la culotte était objet de dispute parce que victime de la concupiscence des femmes et symbole même de l'autorité de l'homme sur la femme ${ }^{26}$. Les versions de la «Lutte pour la culotte » témoignent d'un monde déglingué et placent au fondement du désordre la sexualité insatiable et l'insubordination des femmes, vices qui ne vont pas l'un sans l'autre. Mais l'accent semble désormais définitivement mis sur le second de ces défauts. L'estampe de l'époque moderne ne s'attaque plus directement à la prétention féminine à l'assouvissement charnel; elle s'insurge bien plus contre la domination du sexe féminin sur le masculin, dénoncée à travers la contestation du pouvoir dans le couple conjugal (fig. 7 et fig. 8). Entre le XVII et le XIX ${ }^{e}$ siècle, le lieu de cette revendication s'identifie donc toujours plus avec le ménage. Au XIXe siècle, même si le glissement qui affecte l'objet de la convoitise des femmes conserve une allusion au thème originel, l'estampe a oublié la bataille collective des femmes pour se cantonner dans la scène de ménage autour du pantalon ${ }^{27}$. La violence des relations conjugales doit s'aligner sur le modèle du «couple conversationnel». Que l'image soit dédoublée ou qu'elle présente isolément le contremodèle à éviter, sa leçon est d'abord celle de la conformité sociale.

Les images modernes accordent ainsi à l'inversion des rôles féminin et masculin une place de choix, mais c'est par autant de démonstrations par l'absurde de situations impossibles. Toutefois les mondes à l'envers qui versent dans la folie proposent comme en creux aux couples mal accordés ou désunis une morale de la paix conjugale et de l'ordre familial, ainsi que Natalie Davis l'a suggéré dans son célèbre article « La chevauchée des femmes $»^{28}$.

26 Notons le texte qui accompagne la gravure sur cuivre anonyme de la fin du XVI siècle (fig. 3), où sept femmes s'arrachent une culotte bouffante d'homme tandis qu'un fou s'esquive discrètement: "Traités modestement, amoureuse cohorte,/ Ce membre, car ce n'est pied, teste, épaule ou main/ Pour quy vous vous donnés la bataille si forte,/ Mais le père germeux de tout le genre humain » ; BeaumontMaillet 1984 : 20-21 et fig. 13.

27 Voir les lithographies de Paris, Épinal et Weissemburg in Fuoco Acqua Cielo Terra, 1995 : nº 805 (v. 1860), 807 (v. 1880), 810 (v. 1890), 816 (dernier quart du XIXe s.).

28 Sur le contexte social et l'ambivalence de ces représentations, outre Davis 1979 : 212 ; Niccoli 1981 : 58 ; Matthews Grieco 1997 : 86-87. 


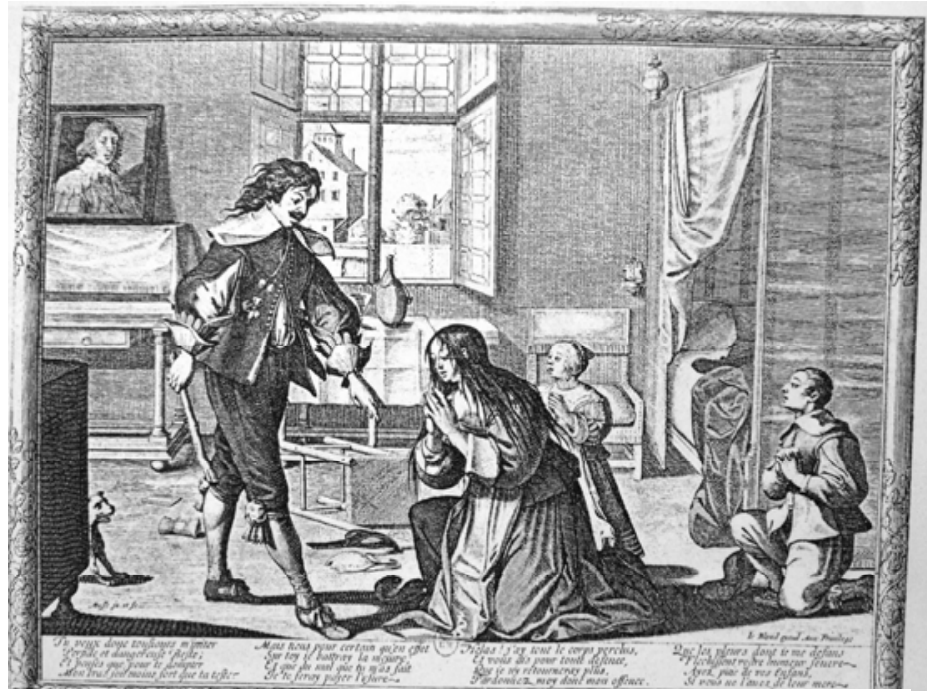

7. Abraham Bosse, Le mari qui bat sa femme. Paris, Leblond, milieu du XVII' $\mathrm{s}$. Paris, BNF (d'après Beaumont-Maillet 1984 : 80, ill. 75).

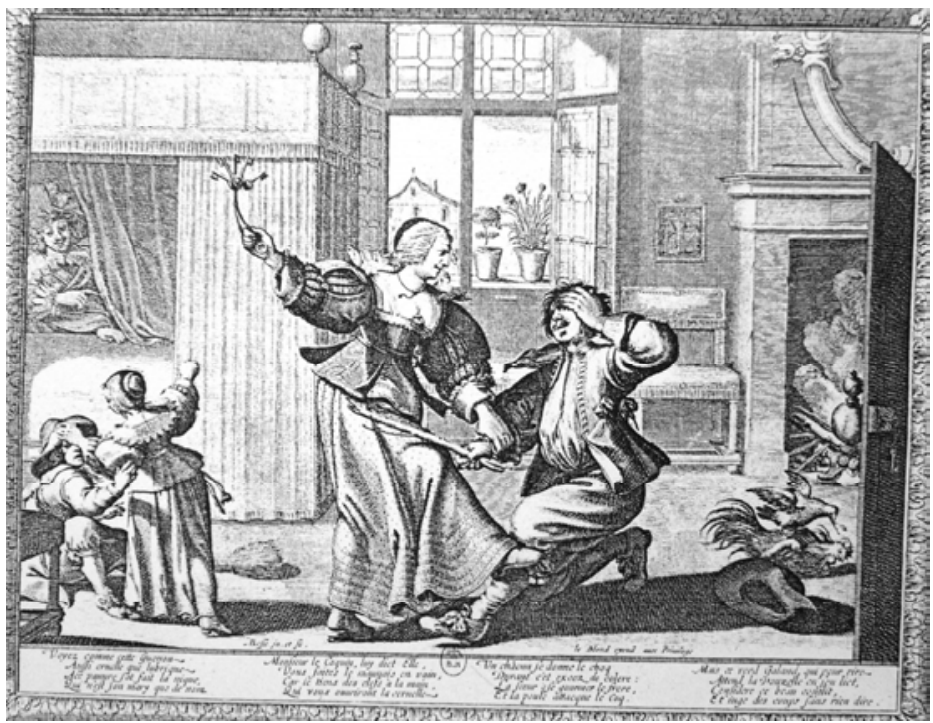

8. Abraham Bosse, La femme qui bat son mari. Paris, Leblond, milieu du XVII ${ }^{e} \mathrm{~s}$. Paris, BNF (d'après Beaumont-Maillet 1984 : 81, ill. 76). 
Lorsque l'amateur d'estampes n'est plus convié à lire le remède au désordre dans les couples d'images dont l'une réaffirme, en contrepoint de l'autre, la juste autorité maritale (fig. 7 et 8 ), il est encore invité à percevoir la bonne manière d'agir dans la profusion d'exemples absurdes aptes à susciter la répulsion, tels le chasseur rôti, le cavalier devenu monture ou le mari filant la quenouille des images d'Épinal.

\section{Bibliographie}

BAGNOLI Alessandro, 2000, «L'albero della fecondità. La scoperta di un raro affresco del 1265 ", in Massa Marittima. L'albero dellà fecondità, Massa Marittima, Comune di Massa Marittima e Soprintendenza per i Beni artistici e Storici di Siena e Grosseto.

BeAumont-Maillet Laure, 1984, La guerre des sexes (XVe-XIXe siècles), Paris, Albin Michel.

Beuningen Hendrik J.E. van \& Ad. Maria Koldeweij (dir.), 1993, Heilig en Profaan, 1000 laatmiddeleeunse insignes wit de collectie H.J.E. van Beuningen, Cothen, Stichting Middeleeuwse religieuze en profane insignes.

BlaschitZ Gertrud, HundsBICHLER Helmut, JaRITZ Gerhard \& Elisabeth VAVRA (Hrsg.), 1992, Symbole des Alltags. Alltag der Symbole. Festschrift für Harry Kübnel, Graz, Akademische Druck- und Verlagsanstalt.

BLOCH R. Howard, 1986, The scandal of the fabliaux, Chicago \& Londres, University of Chicago Press.

BUREAU Pierre, 1995, «La 'Dispute pour la culotte': variations littéraires et iconographiques d'un thème profane (XIII'-XVIe siècles) ", Médiévales, 29, p. 105-129.

CAMILLE Michael, 1992, Image on the edge. The margins of medieval art, Londres, Reaktion Books [trad. fr. de Jean-Claude et Béatrice BONNE, Images dans les marges : aux limites de l'art médieval, Paris, Gallimard, 1997].

-, 1998, «Obscenity and erasure: censorship in medieval illumination of manuscripts ", in Jan M. ZIOLKOWSKI (ed.), Obscenity: social control and artistic creation in the European Middle Ages, Leyde, Brill, p. 139-154.

Contes pour rire. Fabliaux des XIII et XIVe siècles, 1977, trad. Nora SCOTT, Paris, Union générale d'éditions, coll. «10/18».

CotTias Myriam, Downs Laura, KLAPISCH-Zuber Christiane \& Gérard Jorland (dir.), 2010, Le corps, la famille et l'État. Hommage à André Burguière, Rennes, Presses universitaires de Rennes.

DAvIS Natalie Zemon, 1979, "La chevauchée des femmes", in Les cultures du peuple. Rituels, savoirs et résistances an 16 siècle, trad. fr. de Marie-Noëlle BOURGUET, Paris, 
Aubier, p. 210-250. [traduit de "Women on top", in Society and culture in early modern France, Stanford, Stanford University Press, 1975, p. 124-151].

Fabliaux érotiques. Textes de jongleurs des XII et XIII siècles, 1993, édités par Luciano RossI \& Richard STRAUB, Paris, Librairie générale française, coll. «Livre de poche ».

FERzoco George, s.d., «Il murale di Massa Marittima », Toscana Studies, 1.

GRAND-CARTERET John (dir.), 1927-1928, L'bistoire, la vie, les mours et la curiosité par l'image, le pamphlet et le document (1450-1900), Paris, Librairie de la curiosité et des beaux-arts, 5 vol.

HÉBERT Michèle, 1982, Cabinet des estampes. Inventaire des gravures des Écoles du Nord 1440-1550, Paris, BNF.

HOLSTEIN Friedrich Wilhelm Heinrich, 1949, Dutch and Flemish Etchings, Engravings and Woodcuts, ca. 1450-1700, vol. 12, Roosendaal (Neth.), Koninklijke Van Poll.

HuOT Sylvia, 1993, The Romance of the Rose and its medieval readers. Interpretation, reception, manuscript transmission, Cambridge, Cambridge University Press.

JACKOBSOn SchutTe Anne, 1980, «'Trionfo delle donne': tematiche di rovesciamento dei ruoli nella Firenze rinascimentale », Quaderni storici, 44, a. XV, fasc. II, p. 474-496.

JARITZ Gerhard, 1992, "Die Bruoch», in Gertrud BLASCHITZ et al. (Hrsg.), Symbole des Alltags. Alltag der Symbole. Festschrift für Harry Kühnel, Graz, Akademische Druckund Verlagsanstalt, p. 395-416.

Johndon Geraldine \& Sara MatThews Grieco (eds), 1997, Picturing Women in Renaissance and Baroque Italy, Cambridge, Cambridge University Press.

JONES Malcolm, 1994, "Sex and sexuality in late medieval and early modern art », in Daniela Erlach, Marcus Reisenleitner \& Karl VocelKa (Hrsg.), Privatisierung der Triebe. Sexualität in der früben Neuzeit, Francfort/M., Peter Lang, p. 187-304.

KLAPISCH-ZuBER Christiane, 2010, «L'arbre aux galants », in Myriam CoTTIAS, Laura Downs, Christiane KLAPISCH-Zuber \& Gérard JoRLAND (dir.), Le corps, la famille et l'État. Hommage à André Burguière, Rennes, Presses Universitaires de Rennes, p. 117-132.

KRAus Dorothy et Henry, 1986, Le Monde caché des miséricordes; suivi du répertoire de 400 stalles d'église en France, Paris, Éd. de l'Amateur.

LIPPMANN Friedrich, 1886, "Ein italienischer und ein deutscher Kupferstich des XV. Jahrhundert », Jahrbuch der Preussischen Kunstsammlungen, 7, p. 73-81.

LuTZ Eckart Conrad, Johanna THALI, René WETZEL (Hrsg.), 2005, Literatur und $W$ andmalerei. Konventionalität und Konversation, Tübingen, Max Niemeyer.

Matthews Grieco Sara F., 1991, Ange ou diablesse. La représentations de la femme au XVIe siècle, Paris, Flammarion. 
—, 1997, "Pedagogical prints: Moralizing Broadsheets and Wayward Women in Counter Reformation Italy », in Geraldine JOHNDON \& Sara MATTHEWs GrIECO (eds), Picturing Women in Renaissance and Baroque Italy, Cambridge, Cambridge University Press, p. 61-87.

MetKen Sigrid, 1996, Der Kampf um di Hose. Geschlechterstreit und die Macht im Haus. Die Geschichte eines Symbols, Francfort/M. \& New York, Campus Verlag / Paris, Éd. de la Fondation de la Maison des Sciences de l'Homme.

NiCCOLI Ottavia, 1981, «Lotte per le brache. La donna indisciplinata nelle stampe popolari d'ancien régime », Memoria, 2, p. 49-63.

RANDALL Lilian M.C., 1966, Images in the margins of Gothic manuscripts, Berkeley \& Los Angeles, University of California Press.

VEY Horst, 1964, «Der Kampf um die Hosen », Museen in Köln, 3, p. 271-272.

WARBURG Aby, 1998, «Austausch künstlerischer Kultur zwischen Norden und Süden im 15. Jahrhundert », in Aby WARBurG, Gesammelte Schriften, Bd. I, 1: Die Erneuerung des heidnischen Antike, Kulturwissenschaftliche Beiträge zur Geschichte der europäischen Renaissance, Horst BREDERKAMP \& Michael Diers (Hrsg.), Berlin, Akademie Verlag, p. 177-184 et p. 368. [1 re éd. all. par Gertrud BING \& Fritz Rougemont, 1932].

—, 2000 (rééd.), Der Bilderatlas Mnemosyne, Martin WARnke \& Claudia Brink (Hrsg.), Berlin, Akademie Verlag.

WOLTER-VON DEM KNESEBECK Herald, 2005, «Zahm und Will: thematische Spannungsverhältnisse und ihre (topographische) Organisation; die Wandmalereien des Jagdzimmers von Schloss Moss in Eppan », in Eckart Conrad LuTZ, Johanna THALI, René WeTZEL (Hrsg.), Literatur und Wandmalerei. Konventionalität und Konversation, Tübingen, Max Niemeyer, p. 479-519.

ZIOLKOwSKI Jan M. (ed.), 1998, Obscenity: social control and artistic creation in the European Middle Ages, Leyde, Brill.

ZuCKER Mark J. (ed.), 1980, The Illustrated Bartsch, 25, Early Italian Masters, New York, Abaris Books. 